\title{
Análisis de necesidades de metodología docente y actitud de aprendizaje del idioma inglés
}

\author{
Maricela Astrid Hernández Tamacas \\ Maestra en Lingüística Aplicada \\ Docente investigadora, Facultad Multidisciplinaria de Ilobasco, Cabañas \\ Universidad Católica de El Salvador, El Salvador \\ Email: maricela.hernandez@catolica.edu.sv \\ ORCID: https://orcid.org/0000-0002-1754-1802
}

Fecha de recepción: $12-07-2019$

Fecha de aceptación: 22-02-2020

\section{Resumen}

La finalidad de esta investigación fue determinar las necesidades de metodología docente y la actitud de aprendizaje del idioma inglés en los estudiantes de primer año de la Licenciatura en Ciencias de la Educación con Especialidad en Idioma inglés y la Licenciatura en Inglés. Esto con la finalidad de crear objetivos centrados en las características específicas de los estudiantes, que ayuden a potenciar el aprendizaje.

El estudio de tipo exploratorio fue desarrollado con el paradigma cualitativo. Se encuestó a 60 estudiantes de primer año de ambas licenciaturas, utilizando una encuesta con formato de análisis de necesidades. Con esta técnica se encontró que los estudiantes necesitan un mejor tipo de evaluación, especialmente una enfocada en el aprendizaje.

Palabras clave: Metodología docente, actitud de aprendizaje, análisis de necesidades, adecuación curricular, disparidad, opinión docente, opinión del estudiante.

\begin{abstract}
The purpose of this research was to determine the needs of teaching methodology and the learning attitude of the English language in the first year students of the Bachelor of Arts with specialization in English Language and the Bachelor of Arts in English. This in order to create objectives focused on the specific students' characteristics, which help to enhance learning.

The exploratory type study was developed with the qualitative paradigm. 60 first-year students from both degrees were surveyed, using a survey with a needs analysis format. With this technique, it was found that students need a better type of assessment, especially one focused on learning.
\end{abstract}

Key words: Teaching methodology, learning attitude, analysis of needs, curricular adequacy, disparity, teaching opinion, student opinion. 


\section{Introducción}

En contextos de enseñanza aprendizaje, "los maestros comúnmente asumen que sus propios objetivos educativos, que forman su metodología, coinciden con las necesidades e intereses de sus alumnos; lo cual regularmente no es el caso" (Sava, 2012, p. 19). Aunque lo anterior sea una práctica habitual en las evaluaciones docentes de los maestros de inglés, es frecuente que los estudiantes de primer año de estas carreras hagan énfasis en la necesidad de tener una metodología diferente por parte de los docentes; específicamente una enfocada en un aprendizaje competente. Esto mediante: horas extras y personalizadas; más explicaciones y vocabulario, entre otros aspectos.

De acuerdo con Long (2005), hay una noción generalizada en el ámbito de la enseñanza de un segundo idioma; en cuanto a que todo lo que el profesor puede enseñar, en determinada materia y tiempo, el alumno debería de ser capaz de aprenderlo. Gracias a ello, hay una disparidad en lo que los docentes y estudiantes consideran adecuado para el proceso de enseñanza aprendizaje. Por lo tanto, se debe de investigar lo que los estudiantes necesitan respecto a la metodología del maestro y de su propia metodología para el aprendizaje de un idioma.

Para evitar la disparidad entre estudiantes y docentes, debería existir una adecuación curricular enfocada en las necesidades de los primeros. De acuerdo con Ferguson y Jeachild (1999), las adecuaciones curriculares implican el conoci- miento de los estudiantes de sus fortalezas y necesidad para poder diseñar e implementar un currículo. Para hacer una adecuación curricular, el docente debe de conocer a los estudiantes tanto como sea posible; ya que se espera que un excelente maestro: diseñe cursos y aplique métodos de enseñanza apropiados para cumplir con los requerimientos de una población estudiantil (Ramsden, 2007). Para ello se sugiere hacer un análisis de necesidades.

El análisis de necesidades es una aproximación sistemática al estudio del estado del conocimiento; la habilidad, el interés o la actitud de una audiencia definida, o grupo que abarca un tema en particular (McCawley, 2009). Profesionalmente se usa para aprender sobre cuestiones importantes y problemas que enfrenta un público, con la intención de tomar decisiones educadas para diseñar un programa de educación efectivo (McCawley, 2009). La investigación hizo un análisis de las cuestiones que pueden ser problemas para los estudiantes en cuanto a la metodología docente y a su propia metodología de aprendizaje, y que puede llegar a impedir un aprendizaje eficiente.

El estudio evaluó las necesidades de los alumnos en cuanto a sus actitudes hacia el aprendizaje del inglés. Este análisis es el "proceso por el cual se determinan la presencia o ausencia de los factores, condiciones, recursos, servicios y oportunidades de aprendizaje que los estudiantes necesitan para alcanzar sus necesidades educativas, $\mathrm{y}$ objetivos dentro del contexto de la misión de la institución” (Sanva, 2012, p. 23). 
También se analizaron las necesidades pedagógicas, apoyadas en lo propuesto por West (1998) que comprende tres elementos del análisis: de deficiencias, de necesidades de aprendizaje y de medios. Por otra parte, también está el análisis que tiene que ver con las estrategias que los estudiantes emplean para aprender otro idioma, que trata de establecer cómo los estudiantes desean aprender, exponiendo muy poco lo que los estudiantes necesitan aprender (West, 1998). El término análisis de necesidades puede sonar general y sin un sentido propio. Este se utiliza de manera general, pero cada uno de ellos se centra en cuestiones específicas al contexto en el que se desarrolla. A continuación, se presentan dos ejemplos:

- Los análisis de necesidades han sido aplicados alrededor del mundo con diferentes propósitos. En su análisis de necesidades, Boroujeni y Fateme (2013) buscaron determinar si existía una actitud positiva hacia la implementación del método comunicativo con estudiantes iraníes. En esta investigación buscaron determinar información sobre las actitudes, las creencias y la opinión de los estudiantes y maestros.

- Por otra parte, Ulum (2015) aplicó un análisis de necesidades a estudiantes universitarios, desarrollando su capacidad de habla en el idioma inglés, llegando a determinar la necesidad de más materiales y aplicación de actividades auténticas.
Después de realizar un análisis de necesidades, Brown (2009) recomienda que los resultados sean utilizados para generar objetivos claros y precisos del procedimiento a tomar en el aula para que, como sugiere Grier (2005), se vincule el aprendizaje lo más que se pueda con la metodología utilizada por el docente.

Los análisis de necesidades pueden ser aplicados en diferentes ambientes, pero con el mismo objetivo fundamental: determinar el estado de las personas que se ven implicadas en una acción en donde el inglés es un medio necesario de comunicación.

\section{Metodología}

Para determinar la metodología docente y las actitudes de aprendizaje iniciales del Idioma Inglés se desarrolló una encuesta entre los estudiantes de primer año de las carreras. Para ello se aplicó el paradigma cualitativo, a través de un estudio exploratorio con diseño etnográfico.

Los encuestados fueron 60 estudiantes de primer año de Licenciatura en Ciencias de la Educación con Especialidad en Idioma Inglés y Licenciatura en Inglés. El total de la muestra estuvo dividida entre las tres secciones de Inglés Intermedio I del ciclo II - 2019. El procedimiento utilizado incluyo el diseño del instrumento, llenado del instrumento y procesamiento de los datos utilizando Excel.

El instrumento fue un cuestionario estructurado, basado en lo propuesto por Larsen-Freeman y Anderson (2011), en cuanto a su apartado de 
la teoría de enseñanza y aprendizaje comunicativo. El cuestionario constó de dos secciones: ña primera, enfocada en las necesidades de actitudes de aprendizaje iniciales de los estudiantes de primer año; y la segunda, en las necesidades de metodología docente. En cada una de las secciones se hicieron conjuntos de cinco aspectos relacionados que los estudiantes debían leer y marcar como el más importante, asignándole una ponderación de 5; para luego degradar número por número hasta llegar al menos importante que marcarían con un 1 . La encuesta tuvo once diferentes conjuntos que fueron analizados por los estudiantes.

\section{Resultados}

\section{Sección I}

La sección uno del cuestionario se refirió a las actitudes generales que los estudiantes necesitan para aprender inglés al inicio de sus estudios. Este se dividió en cinco conjuntos. A continuación se presenta el descriptor para cada uno de ellos:

\section{a. Características personales}

En este conjunto, la preparación para aprender algo nuevo y el compromiso con el propio aprendizaje, reflejado en una actitud de respeto, obtuvo un $25 \%$. Igual porcentaje obtuvo el compromiso y perseverancia en el aprendizaje, sin mostrarse aburrido o desinteresado (25\%), El conocimiento general alcanzó un 20\%; la madurez y dominio, así como el evitar distracciones con los compañeros logró un
$18 \% \mathrm{y}$; finalmente, la creatividad en general se agenció un $12 \%$.

\section{b. Interacción en la clase}

En este caso, prestar atención y concentrarse, no distrayéndose con el teléfono u otros objetos consiguió ser la necesidad más importante para el aprendizaje (23\%). Trabajar grupalmente y colaborar con los compañeros alcanzó el 21\%. Otro aspecto que también obtuvo similar porcentaje (21\%) fue participar en la clase con facilidad y sin temor; mientras que el trabajar individualmente con efectividad obtuvo el $15 \%$.

\section{c. Hábitos de estudio}

Esta sección se refirió a los hábitos de estudio que son necesarios para aprender eficientemente, entre ellos: distribuir su estudio por días, siendo parte de su rutina diaria (23\%); seguido por el tomar notas de alta calidad para estudiar luego (21\%). Conocer diversas formas de estudiar un material obtuvo el 20\%; y reconocer cuánto deben estudiar para obtener buenas notas, dependiendo de sus habilidades personales, logró un 19\%. El aspecto que menos porcentaje obtuvo fue el tener grupos de estudio con un $17 \%$.

\section{d. Disposición hacia las tareas}

Aquí, estudiar con suficiente tiempo consiguió el 21\%, convirtiéndose en la necesidad que predomina en el conjunto. Saber cómo el estudiante aprende mejor y utilizar esto como herramienta efectiva para su aprendizaje obtuvo el $21 \%$. Los siguientes aspectos fueron 
tener una precepción positiva sobre las tareas y verlas como una manera de aprender (20\%); estar generalmente motivados a hacer las tareas (19\%); mientras que utilizar recursos tecnológicos para estudiar alcanzó el porcentaje más bajo $(18 \%)$.

\section{e. Nivel educativo deseado}

Este giró alrededor del nivel que se debería tener al iniciar los estudios. Los niveles eran: 1 = nada; 2 = poco; $3=$ eficiente y $4=$ fluido. De acuerdo a los estudiantes, el nivel deseado de vocabulario; comprensión lectora, comprensión auditiva; de escritura y gramática, así como la fluidez oral debe de ser de 2.7.
Se muestra un cuadro resumen de la sección uno del cuestionario, conforme el nivel de importancia otorgado (ver figura 1).

\section{Sección II}

La sección dos del cuestionario trató sobre aspectos de docencia y pedagogía que los estudiantes necesitan para aprender inglés al inicio de sus estudios. A continuación, se presenta cada uno de los seis conjuntos de esta sección:

\section{a. Generalidades de la clase}

Dentro de este apartado, la necesidad de que el docente cree una atmósfera positiva y buenas relaciones con los estudiantes obtuvo un $24 \%$; seguido de la necesidad de que el docente ten-

\begin{tabular}{|l|l|c|}
\hline \multicolumn{1}{|c|}{ Conjunto } & \multicolumn{1}{|c|}{ Descriptor } & Importancia \\
\hline $\begin{array}{l}\text { Características } \\
\text { personales }\end{array}$ & $\begin{array}{l}\text { - Prepararse para aprender algo nuevo. } \\
\text { Comprometerse con el propio aprendizaje, reflejado en una } \\
\text { actitud de respeto. }\end{array}$ & 1 \\
\hline $\begin{array}{l}\text { Interacción en } \\
\text { la clase }\end{array}$ & $\begin{array}{l}\text { - Prestar atención y concentrarse. } \\
\text { Evitar distraerse con el teléfono y objetos. }\end{array}$ & 2 \\
\hline $\begin{array}{l}\text { Hábitos } \\
\text { de estudio }\end{array}$ & - Distribuir su estudio por días, siendo parte de su rutina diaria. & 3 \\
\hline $\begin{array}{l}\text { Disposición ha- } \\
\text { cia las tareas }\end{array}$ & - Estudiar con suficiente tiempo. & 4 \\
\hline $\begin{array}{l}\text { Nivel educati- } \\
\text { vo deseado }\end{array}$ & $\begin{array}{l}\text { Debería tener un nivel del 27\% de conocimiento en el idio- } \\
\text { ma inglés al entrar a la carrera. }\end{array}$ & 5 \\
\hline
\end{tabular}

Figura 1. Actitudes estudiantiles necesarias para el aprendizaje de inglés. 
ga una habilidad alta en el inglés (23\%). Que el docente sea dinámico y creativo obtuvo un $20 \%$. Para los encuestados, el hecho que las clases tengan un enfoque comunicativo tiene un $17 \%$, y que el docente hable solamente en inglés obtuvo un $16 \%$.

\section{b. Organización de las clases}

En este conjunto, dos aspectos fueron tomados como más importantes: para un $80 \%$ de los encuestados es recomendable ver un tema por varios días hasta que se comprenda. Que la clase esté bien estructurada y tenga una secuencia lógica; y que las actividades estén conectadas tienen un $24 \%$. Otro aspecto considerado fue que, cada clase tenga una rutina con actividades creativas que impulsen el aprendizaje alcanzado (21\%). Que cada clase tenga un objetivo claro consiguió un 19\%, y el que menos porcentaje consiguió (12\%) fue la necesidad de cubrir la mayor cantidad de temas posibles durante el semestre.

\section{c. Lenguaje utilizado durante la clase}

Acá el aspecto con mayor porcentaje fue que el profesor debe dar a los estudiantes estrategias para utilizar el lenguaje, tanto dentro como fuera del salón de clases (24\%); seguido de la necesidad del docente de utilizar el español para dar explicaciones y dejar puntos claros (23\%). Motivar e impulsar a los estudiantes a hablar solamente en inglés obtuvo un $22 \%$; y que el docente se enfoque en impartir clases con mucha producción consiguió un $16 \%$. Por último, que el lenguaje que se utilice en el salón esté altamente contextualizado obtuvo un $15 \%$.

\section{d. Instrucciones}

De acuerdo con el $25 \%$ de los encuestados, las instrucciones deben ser claras para que los estudiantes sean capaces de seguirlas. El factor que las explicaciones de gramática sean compresibles se agenció un 23\%; y que las instrucciones tengan un alto porcentaje de ejemplificación y explicación contó con un 19\%. Por su parte, formar rutinas para ayudar a los estudiantes a seguir las indicaciones alcanzo un 17\%; y que se entrene intensivamente a los estudiantes para que comprendan las instrucciones y explicaciones, obtuvo un $16 \%$.

\section{e. Materiales de clase}

Para los encuestados, que los materiales estén adecuados al nivel de los estudiantes, y que a través de ellos puedan avanzar de nivel es importante (24\%); seguido de que el docente utilice una variedad de materiales para impartir la clase $(21 \%)$. Por otra parte, el $21 \%$ de los encuestados manifestó la necesidad de que el docente utilice una variedad de técnicas con los materiales para impartir la clase. Por último, que los materiales estén bien diseñados llegando a ser personalizados obtuvo un $14 \%$.

\section{f. Evaluación}

Los encuestados valoraron con un $27 \%$ la importancia que los exámenes y la evaluación tengan un enfoque en el aprendizaje, dejándole saber a los estudiantes qué deben reforzar. Hacer evaluaciones orales de forma frecuente (por lo menos una cada semana), acompañadas de una apropiada retroalimentación, obtuvo un $21 \%$. 
Los participantes puntuaron con un $20 \%$ el evaluar las cuatro macro habilidades: hablar, escribir, comprensión lectora y comprensión auditiva. También señalaron que los exámenes y las evaluaciones deben ser diseñados para medir, apropiadamente, las habilidades de los estudiantes con un sistema en el que la nota pueda obtenerse sin problema (18\%). Finalmente, un 14\% de los involucrados aseguró que se debe evaluar usando la plataforma de la universidad, dando espacio para prácticas y respectiva examinación.

En la figura 2, se presenta el cuadro resumen de los aspectos más importantes de cada uno de los seis conjuntos de esta sección.

\section{Discusión}

Para el aprendizaje del idioma inglés, entre las características generales que los estudiantes más necesitan, se encuentran: la preparación para aprender algo nuevo, el compromiso y la perseverancia; así como también, la capacidad de prestar atención. En cuanto a los hábitos de estudio, lo primordial es distribuir el estudio por días y hacerlo parte de la rutina del estudiante. Aunado a esto, se necesita que el alumno estudie con suficiente tiempo.

Un aspecto significativo que puede llegar a determinar el aprendizaje de un lenguaje es el nivel de inglés que los encuestados creen que se debe tener al iniciar los estudios. Ellos manifestaron que no se sugiere iniciar la carrera con un nivel cero de conocimiento en las diferentes áreas. Señalaron que se debe tener un mínimo de conocimiento en las áreas de: vocabulario, gramática y escritura; comprensión auditiva y comprensión lectora. Ante esto, se recomienda un curso propedéutico intensivo de inglés, antes de iniciar la carrera. Ello aseguraría que los estudiantes inicien con el nivel recomendado por los encuestados dentro de esta investigación.

Los participantes del estudio sugieren a los docentes en cuanto a su metodología, que se debe tener una buena relación con sus estudiantes, generando un ambiente de confianza. También indicaron la necesidad de ver un tema por varios días hasta que se entienda a un $80 \%$. Esto aunado a la necesidad de que la clase esté bien estructurada y con secuencia lógica. El profesor debe dar al estudiante estrategias para utilizar el lenguaje, tanto dentro como fuera del aula; además, las instrucciones deben ser claras. Es responsabilidad de los docentes adecuar los materiales al nivel de los estudiantes, a fin de avanzar en su propio nivel. Por tanto, es ideal que los exámenes y las evaluaciones tengan un enfoque en el aprendizaje.

Una de las cuestiones particulares de este análisis de necesidades fue que, en promedio, hubo un puntaje de ocho puntos de diferencia entre la más y la menos apremiante. Esto podría interpretarse como que la mayoría de los aspectos presentados son significativos. Pero, según, Sanva (2012), los análisis de necesidades sirven para determinar la presencia o ausencia de todo aquello que sea necesario para facilitar el aprendizaje. Por tanto, el hecho que haya pocos puntos de diferencia entre ítems de los con- 


\begin{tabular}{|c|l|c|}
\hline Conjunto & \multicolumn{1}{|c|}{ Descriptor } & Importancia \\
\hline Evaluación & $\begin{array}{l}\text { - Exámenes y la evaluación deben tener un enfoque en el aprendizaje. } \\
\text { - Hacer saber a los estudiantes qué deben reforzar. }\end{array}$ & 1 \\
\hline Instrucciones & $\begin{array}{l}\text { Las instrucciones deben ser claras para que los estudiantes } \\
\text { sean capaces de seguirlas. }\end{array}$ & 2 \\
\hline $\begin{array}{c}\text { Generalidades } \\
\text { de la clase }\end{array}$ & $\begin{array}{l}\text { Que el docente cree una atmósfera positiva dentro del salón } \\
\text { - Que el docente tenga buenas relaciones con los estudiantes. }\end{array}$ & 3 \\
\hline $\begin{array}{c}\text { Organización } \\
\text { de la clase }\end{array}$ & $\begin{array}{l}\text { porcentaje del 80\%. } \\
\text { Clase bien estructurada y con secuencia lógica } \\
\text { - Conexión entre las actividades. }\end{array}$ & 4 \\
\hline $\begin{array}{c}\text { Lenguaje uti- } \\
\text { lizado durante }\end{array}$ & $\begin{array}{l}\text { Que el docente brinde a los estudiantes estrategias para utili- } \\
\text { zar el lenguaje, tanto dentro como fuera del salón de clases. }\end{array}$ & 5 \\
\hline $\begin{array}{c}\text { Materiales } \\
\text { de clase }\end{array}$ & $\begin{array}{l}\text { Materiales adecuados al nivel de los estudiantes; y a través } \\
\text { de ellos poder avanzar de nivel. }\end{array}$ & 6 \\
\hline
\end{tabular}

Figura 2. Docencia y pedagogía para el aprendizaje del inglés.

juntos dentro de la encuesta en general puede significar la ausencia de los factores que facilitan el aprendizaje. Esto lleva a considerar una perspectiva integral, que integre la mayoría de los aspectos tomados en cuenta para impulsar el aprendizaje.

De todos los aspectos valorados, el que las evaluaciones tengan un enfoque hacia el aprendizaje fue el que más despuntó. En cuanto a esto, hay que recordar lo que McCawley (2009) expone respecto a los análisis de necesidades, en donde señala que estos ayudan a determinar problemas que los estudiantes tienen. Al escoger este ítem, los encuestados señalan que las evaluaciones no están centradas en el aprendizaje, representándoles un problema sensible. El señalamiento de este ítem es congruente con el porcentaje relacionado al factor de exigir la mayor cantidad de tema posibles por semestre. Esto lleva a concluir que, si se está enfocado en el aprendizaje, entonces el currículo se debería adecuar a cubrir una determinada cantidad de temas.

En resumen, se espera que un excelente maestro diseñe cursos y aplique métodos de enseñanza adecuados para cumplir con los requerimientos de una población estudiantil (Ramsden, 2007). Todo esto se sugiere en pro de acciones que ayuden a crear objetivos claros para el aprendizaje, en donde este se 
vincule lo más posible, tal como lo aconsejan Brown (2009) y Grier (2005).

Este diseño para los maestros de primer año de la Licenciatura en Ciencias de la Educación con Especialidad en Idioma Inglés y la Licenciatura en Inglés puede ser sustentado por el análisis de necesidades presentado en este artículo. Esto, además de incrementar la calidad académica, también sirve para que los estudiantes conozcan aquellas características que les ayudarán en su proceso de aprendizaje.

\section{Referencias}

Altschuld, J. W. y Witkin, B. R. (2000). From needs assessment to action: Transforming needs into solution strategies. New York: Sage.

Barbazette, J. (2006). Training needs assessment: Methods, tools, and techniques. New York: J. Wiley \&s Sons.

Boroujeni, S. A. y Fateme, M. F. (2013). A needs analysis of English for specific purposes (ESP) course for adoption of communicative language teaching: A case of Iranian first-year students of educational administration. International Journal of Humanities and Social Science Invention, 2 (6), 35-44.

Brown, J.D. (1995). The elements of language curriculum: A systematic approach to program development. Boston, Massachusetts: Heinle \& Heinle Publishers.

Dudley-Evans, T. y St. John, M. (1998). Developments in ESP: A multi-disciplinary approach. Cambridge: Cambridge University Press.

English, L. M. (Ed.) (2005). International encyclopedia of adult education. New York: Palgrave Macmillan.

Ferguson y Jeanchild (1999). ¿Cómo poner en práctica las decisiones curriculares? En: Stainback y Stainback. (Comp.) (1999). Aulas Inclusivas. Madrid: Narcea. (pp. 178-194).

Grier, A.S. (2005). Integrating needs assessment into career and technical curriculum development. Journal of Industrial Teacher Education. 42 (1), pp.59-66

Gupta, K. (2007). A practical Guide to Needs Assessment. John Wiley \& Sons, Inc. Pfeiffer. San Francisco.

Hutchinson T. y Waters A. (1987). English for Specific Purposes: A Learning-Centered Approach. Cambridge. 
Iwai, T.; Kondo, K.; Limm, S. J. D.; Ray, E. G.; Shimizu, H. y Brown, J. D. (1999). Japanese language needs analysis. Recuperado de http://www.nflrc.hawaii.edu/Networks/NW13/NW13.pdf.

Jasso-Aguilar, R. (2005). Sources, methods and triangulation in needs analysis: a critical perspective in a study of Waikiki hotel maids. In Long. H. M (Eds), Second Language Needs Analysis. Cambridge University Press. Cambridge.

Kettner, P. M.; Moroney, R. y Martin, L. (1999). Designing and Managing programs: An effectiveness-based approach (2nd ed.). New York: Sage.

Larsen-Freeman, D. y Anderson, M. (2011). Techniques and Principles in Language Teaching. Oxford. Oxford University Press.

Long, M.H. (2005a). Methodological issues in learner needs analysis. In M.H. Long (Ed.). Second language needs analysis (pp. 19-76). Cambridge: Cambridge University Press.

Long, M.H. (2005b). Overview: A rationale for needs analysis and needs analysis research. In M.H. Long (Ed.), Second language needs analysis (pp. 1-16). Cambridge: Cambridge University Press.

Mackay, R. y Mountford, A. (Eds.). (1978). English for specific purposes. London: Longman

McCaslin, N. L. y Tibezinda, J. P. (1996). Chapter 5: Assessing target group needs. In B. E. Swanson, R. P. Bentz y A. J. Sofranco (Eds.), Improving agricultural extension: A reference manual. Ramsden, P. (2007). Learning to Teach in Higher Education. Londres/Nueva York. Routledge Falmer. Sava, S. (2012). Needs analysis and program planning in adult education. In Budrich, B (Eds.), Study grades in adult education. Opladen. Germany.

Ulum, O. G. (2015). A needs analysis study for preparatory class EFL students. European Journal of English Language Teaching.

West, R. (1998). ESP-State of the art. Recuperado de: www.man.ac.uk/CELSE/esp/west.htm 